\title{
Breast Cancer Surgery in the COVID-19 Pandemic: Validation of a Preventive Program for Patients and Health Care Workers
}

\author{
PIERO FREGATTI ${ }^{1,2}$, MARCO GIPPONI $^{2}$, MARIA GIACCHINO ${ }^{1}$, MARCO SPARAVIGNA ${ }^{1}$, \\ RAQUEL DIAZ ${ }^{2}$, EMANUELA FIORAVANTI ${ }^{1}$, CHIARA CORNACCHIA ${ }^{1}$, BENEDETTA CONTE ${ }^{3,4}$, \\ MATTEO LAMBERTINI ${ }^{3,4}$, GABRIELE ZOPPOLI $^{3,5}$, FEDERICA MURELLI $^{1,2}$, MARIA LUISA TONI $^{6}$, \\ MARIA TERESA CALABRO ${ }^{1}$, LINA ORSINO $^{6}$ and DANIELE FRIEDMAN ${ }^{1,2}$ \\ ${ }^{1}$ Department of Surgical Sciences and Integrated Diagnostic (DISC), \\ School of Medicine, University of Genoa, Genoa, Italy; \\ ${ }^{2}$ Breast Surgery Clinic, IRCCS Ospedale Policlinico San Martino, Genoa, Italy; \\ ${ }^{3}$ Department of Internal Medicine and Medical Specialties (DiMI), \\ School of Medicine, University of Genoa, Genoa, Italy; \\ ${ }^{4}$ Department of Medical Oncology, U.O.C Clinica di Oncologia Medica, \\ IRCCS Ospedale Policlinico San Martino, Genoa, Italy; \\ ${ }^{5}$ Internal Medicine and Oncology Unit, IRCCS Ospedale Policlinico San Martino, Genoa, Italy; \\ ${ }^{6}$ Direction Health Professions (DAPS), IRCCS Ospedale Policlinico San Martino, Genoa, Italy
}

\begin{abstract}
Background/Aim: The perspective validation of a selective approach in patients undergoing breast cancer surgery was performed in order to assess whether patients as well as Health Care Workers (HCWs) were exposed to any undue risk of COVD-19 infection. Patients and Methods: From March $9^{\text {th }}$ to June $9^{\text {th }} 2020,207$ patients were phonetriaged by a dedicated Breast Care Nurse; a patient-tailored program was adopted with the aim of avoiding hospitalization of SARS-CoV-2 symptomatic patients, with a careful prioritization of surgical procedures according to specific disease features. Results: Two hundred and three out of 207 patients underwent operation; seven patients were temporarily excluded because they tested positive at phone triage $(n=3)$, or in-hospital triage ( $n=3)$; another asymptomatic patient with negative NP swab tested IgM Ab-positive so that surgery was re-scheduled two weeks later. Four patients had no surgery; one of them was reconsidered for neoadjuvant chemotherapy (NAC) after testing positive at phone triage; three patients were excluded because they were already hospitalized for COVID-19. Overall, mean in-hospital stay was 2.2 days $( \pm S D$, $0.7)$ and, after hospital discharge, no patient required re-
\end{abstract}

This article is freely accessible online.

Correspondence to: Marco Gipponi, MD, Breast Surgery Clinic, Ospedale Policlinico San Martino, L.go R. Benzi, 10, 16132 Genoa, Italy. Tel: +39 3470530446, e-mail: marco.gipponi@hsanmartino.it

Key Words: COVID19, breast cancer, clinical management. admission. Conclusion: This preventive program avoided any COVID-19 infection among patients and HCWs, so that an elective breast cancer surgical procedure can be safely and timely pursued without affecting the oncologic outcome.

On June $9^{\text {th }}, 235,561$ cases of Coronavirus disease-2019 (COVID-19) infection were registered in Italy, with 32,872 active patients, and 34,043 related-deaths; worldwide, epidemiologic data indicated 7,322,817 cases of COVID-19 infection with 416,678 related-deaths (1-3). On these grounds, health-care systems have consequently modified their practice in order to preserve and optimize the resource allocation while minimizing the risk of COVID-19 infection among patients and Health Care Workers (HCWs).

Preliminary results of a perspective clinical study regarding the evaluation of a patient-tailored program in the COVID-19 era at the Breast Surgery Clinic of "IRCCS Ospedale Policlinico San Martino" in Genoa aimed at the: 1) pre-hospitalization screening by means of a "Patient Screening Flow-chart", in order to avoid the hospitalization of symptomatic COVID-19 patients, and 2) prioritization of the surgical procedures according to specific disease features, which have been already published (4-5). The primary aim was to avoid any delay of oncologic treatment, while sparing the patient as well as HCWs of any undue risk of COVD-19 exposure. No demonstrated COVID-19 infection among patients and HCWs was detected during the first month of this dedicated surgical activity, so the safety of this health-care screening program and its feasibility in a high-volume breast unit were confirmed. Herein, the 
Table I. Guidelines for patient selection.

\begin{tabular}{ll}
\hline Treating group patients & Deferred group patients \\
\hline $\begin{array}{l}\text { Neoadjuvant patients finishing treatment (any T any N stages) } \\
\text { Clinical Stage T1/2 and N0 ER pos/PgR pos/HER2 negative tumors } \\
\text { in patients }<50 \text { years old }\end{array}$ & $\begin{array}{l}\text { Benign lesions (fibroadenoma, nodules, } \text { etc.) } \\
\text { Duct excision (mostly in case of suspected intraductal papillomas) }\end{array}$ \\
$\begin{array}{l}\text { Clinical Stage T1 N0 Triple negative or HER2 positive patients } \\
\text { Suspect biopsies likely to be malignant, B4 }\end{array}$ & $\begin{array}{l}\text { Doubtful biopsies likely to be benign, B3 } \\
\text { Pxcision of malignant recurrence }\end{array}$ \\
$\begin{array}{l}\text { Clinical Stage T1/T2 N0 ER pos/PgR pos/HER2 negative tumors in } \\
\text { pe-excisionts }>50 \text { years old, where neoadjuvant hormonal treatment in given } \\
\text { cTisN0 lesions (in particular if nodular presentation, G3 or extensive }\end{array}$ & $\begin{array}{l}\text { Tumors responding to neoadjuvant chemotherapy } \\
\text { Clinical Stage N+ and any T, redirected to neoadjuvant therapy }\end{array}$
\end{tabular}

area of microcalcifications)

Uncertain malignant potential tru-cut biopsy, B3*

*Included into the Treating group from May $2^{\text {nd }} 2020$.

validation of this selective clinical and epidemiological approach on a wider perspective sample of patients is reported coupled with a longer follow-up assessment.

\section{Patients and Methods}

The Patient Screening Flow-chart and Patient Selection Groups have been previously described; case-tailored flow-charts were developed to screen breast cancer patients for COVID-19 symptoms (fever, cough and/or respiratory symptoms) from March $9^{\text {th }}$ to June $9^{\text {th }}$ 2020. Three distinct settings were considered: home, hospitalization, and post-discharge triage (5-9). According to the American College of Surgeons guidelines, patients were divided into two groups: the "Treating Group Patients", corresponding to patients with an actual risk of impaired survival if surgery was delayed for more than three months, and the "Deferred Group Patients", including patients who might have a delay of the surgical procedure until resolution and/or mitigation of COVID-19 pandemic (Table I) (10). The preferred surgical option was breast-conserving surgery (BCS), whenever possible; total mastectomy, either NippleSparing Mastectomy (NSM) or simple mastectomy with reconstruction, was performed in the remaining patients. Clinical charts were weekly discussed by video-conferencing by the Breast Cancer Management Team. Clinical information was already specified and signed in the standard Privacy Module of each patient so that no Regional Ethics Committee was required.

\section{Results}

From March $9^{\text {th }}$ to June $9^{\text {th }} 2020,207$ patients had a phone interview by a dedicated Breast Care Nurse. Seven patients were transiently excluded from the scheduled operation because they tested positive either at the phone or in-hospital triage; three of them were excluded at phone triage and they had their operation re-scheduled due to COVID-19 symptoms; three other patients were excluded at in-hospital triage due to nasopharyngeal (NP) swab positivity $(n=2)$ even if they were completely asymptomatic with IgM-negative antibodies (Ab); they were referred to the Public Health Office for daily tele-
Table II. Preoperative criteria for the selection of the "Treating Group Patients".

\begin{tabular}{lrr}
\hline Preoperative breast diagnosis & \multicolumn{2}{c}{ Patients } \\
\cline { 2 - 3 } & N. & $\%$ \\
\hline Completed neoadjuvant chemotherapy regimen & 23 & 11.4 \\
cT1/2N0, ER pos/PgR pos/HER2-negative & 78 & 38.4 \\
patients <50 years old & & \\
cT1N0 "Triple-negative" or HER2-positive patients & 32 & 15.8 \\
Suspected tru-cut biopsy (B4) & 14 & 6.9 \\
Local recurrence & 3 & 1.5 \\
Re-excision surgery & 5 & 2.5 \\
cTisN0, ER positive and negative & 22 & 10.8 \\
Doubtful tru-cut biopsy (B3)* & 26 & 12.8 \\
Total & 203 & 100.0 \\
\hline
\end{tabular}

*Included into the Treating group from May $2^{\text {nd }} 2020$.

monitoring, and surgery was re-scheduled two weeks later, according to WHO recommendations (11-12). The last patient was asymptomatic although she showed increased levels of IgM Ab with negative NP swab; hence, she was managed by the Public Health Office Team, and surgery was re-scheduled two weeks later as soon as a second negative NP swab was obtained (13-14).

Four patients did not undergo surgery; one of them was reconsidered for neoadjuvant chemotherapy (NAC) after testing positive at phone triage, and three patients were excluded because they were already hospitalized for COVID19, all of whom later died due to COVID-19. Hence, 203 patients underwent elective breast surgery, 183 (90\%) of whom were selected by our Breast Unit, while 20 (9.8\%) came from other Regional Breast Units.

The "Treating Group Patients" criteria are reported in Table II; from May $2^{\text {nd }} 2020$ patients with doubtful tru-cut biopsy lesions (B3) were included into the "Treating Group 
Fregatti et al: Breast Cancer \& COVID-19 Pandemic

Table III. Distribution of patient by pathologic diagnosis, stratified by selecting Center.

\begin{tabular}{|c|c|c|c|c|c|c|}
\hline \multirow[t]{2}{*}{ Pathologic diagnosis } & \multicolumn{2}{|c|}{ Breast Clinic - Genoa } & \multicolumn{2}{|c|}{ Regional Breast Units } & \multicolumn{2}{|c|}{ Total } \\
\hline & $\mathrm{N}$. & $\%$ & $\mathrm{~N}$ & $\%$ & $\mathrm{~N}$. & $\%$ \\
\hline Invasive Breast Cancer & 127 & $(69.4)$ & 14 & $(70.0)$ & 141 & $(69.4)$ \\
\hline DCIS & 22 & $(12.0)$ & - & 22 & $(10.8)$ & \\
\hline B4 & 13 & $(7.0)$ & 1 & $(5.0)$ & 14 & (6.9) \\
\hline $\mathrm{B} 3 *$ & 21 & $(11.5)$ & 5 & $(25.0)$ & 26 & $(12.8)$ \\
\hline Total & 183 & $(100.0)$ & 20 & (100.0) & 203 & $(100.0)$ \\
\hline
\end{tabular}

DCIS: Ductal Carcinoma in situ; B4: suspected tru-cut biopsy; B3: doubtful tru-cut biopsy, *included into the Treating group from May $2^{\text {nd }} 2020$.

Table IV. Surgical procedure stratified by preoperative histology.

\begin{tabular}{|c|c|c|c|c|c|c|}
\hline \multirow[b]{2}{*}{ Procedures } & \multirow{2}{*}{$\begin{array}{c}\text { Invasive Breast Cancer } \\
\text { N. pts }\end{array}$} & \multirow{2}{*}{$\begin{array}{l}\text { DCIS } \\
\text { N.pts }\end{array}$} & \multirow{2}{*}{$\begin{array}{l}\text { B4 } \\
\text { N. pts }\end{array}$} & \multirow{2}{*}{$\begin{array}{l}\text { B3 } \\
\text { N.pts }\end{array}$} & \multicolumn{2}{|c|}{ Total } \\
\hline & & & & & $\mathrm{N}$. & $\%$ \\
\hline BCS+SLNB & 76 & - & - & & 76 & 37.4 \\
\hline Lumpectomy & & 16 & 14 & 26 & 56 & 27.6 \\
\hline NSM+SLNB+reconstruction* & 29 & 6 & - & & 35 & 17.2 \\
\hline Simple Mastectomy & 20 & - & - & & 20 & 9.8 \\
\hline $\mathrm{BCS}+\mathrm{SLNB}+\mathrm{ALND}$ & 8 & - & - & & 8 & 3.9 \\
\hline BCS+ALND & 4 & - & - & & 4 & 1.9 \\
\hline Bilateral NSM+SLNB+reconstruction & 4 & - & - & & 4 & 1.9 \\
\hline Total & 141 & 22 & 14 & 26 & 203 & 100.0 \\
\hline
\end{tabular}

BCS: Breast-conserving surgery; DCIS: ductal carcinoma in situ; B4: highly suspicious tru-cut biopsy; B3: doubtful tru-cut biopsy; SLNB: sentinel lymph-node biopsy; ALND: axillary lymph node dissection; *homolateral reconstruction.

Patients", as well. The pathologic diagnosis of scheduled operations stratified by the Referral Centre are reported in Table III, most patients having a preoperative diagnosis of invasive breast cancer (141 out of 203 patients, 69.4\%).

Surgical procedures are reported in Table IV: 144 out of 203 patients $(70.4 \%)$ underwent BCS; 39 patients $(19.2 \%)$ had NSM with breast reconstruction, and the remaining 20 patients $(9.8 \%)$ had simple mastectomy. The mean inhospital stay was equal to 2.2 days $( \pm \mathrm{SD}, 0.7)$. No readmission was required after hospital discharge, all of them being managed in the Outpatient Clinic. Finally, as regards the patient's attitude toward the disease, 15 out of 203 patients $(7.4 \%)$ were more afraid of COVID-19 than of their breast cancer, as suggested by a binary question given to each patient after one month from surgery.

\section{Discussion}

In the present experience, a specific preventive strategy was conceived according to national and international recommendations with the aim of performing a preoperative COVID-19 screening, thus avoiding any impairment of breast cancer patient's outcome related to a delay of treatment planning, while preserving our clinical and surgical work-force. Seven out of 207 patients (3.3\%) were temporarily suspended from the scheduled operation after the "Telephone Triage", six of whom because of COVID-19 symptoms (3 patients) or hospitalization due to COVID-19 infection (the other 3 patients), while one received NAC after oncologic re-assessment. All patients that had been hospitalized for COVID-19 disease died. Thus, most of the patients underwent safe surgical procedures without any undue post-operative complication and without any demonstrated COVID-19 infection among patients and HCWs, notwithstanding the reduced availability of operating theatres and Anaesthetists. Hence, 163 patients with a preoperative diagnosis of breast cancer $(80.3 \%)$ had the scheduled operation, 141 of whom for invasive cancer (69.4\%) and 22 for ductal carcinoma in situ (DCIS) (10.8\%). Worth of note, four of the latter group had invasive cancer at post-operative histological examination.

The rate of female patients coming from other Regional Breast Units was rather low (20 out of $203=9.8 \%$ ), and this might be related to: 1) a delayed recruitment of patients coming from the other Breast Units; 2) doubts regarding the oncologic management by another team, far from home, 
while still hoping for a rather quick resolution of COVID-19 pandemic; 3 ) the fear of logistic obstacles for accomplishing adjuvant treatments (such as adjuvant medical treatment or radiation therapy) and, last but not least, the fear of acquiring COVID-19 infection during hospitalization (5).

In-hospital stay was strictly limited to the least for avoiding any unnecessary overload to health-care structures, with a mean length of stay of approximately one day more as expected in the pre-COVID pandemic due to the preventive measures that were required. All patients were regularly discharged with breast and/or axillary drainages as soon as they were clinically stable, and a systematic home tele-monitoring was developed and supervised by dedicated Breast Care Nurses.

Certainly, the most relevant issue regards the selection of the "Treating Group Patients". In the present experience, patients with doubtful malignant potential tru-cut biopsy lesions (B3) were eligible for surgery. Hence, 26 patients $(12.8 \%)$ with preoperative B3 diagnosis were surgically treated, and seven of them $(26.9 \%)$ had a post-operative diagnosis of invasive cancer. This category was added to the eligible group of patients for surgery due to the delay in mammography screening from March $9^{\text {th }}$ to June $9^{\text {th }}$. Hence, less breast cancer diagnoses were collected, and in less than two weeks, patients with a definite preoperative diagnosis of breast cancer could undergo surgical treatment; thanks to this addendum, these seven patients had a prompt surgical therapy thus, speeding the treatment planning and execution.

A great attention regards the possibility to choose, even in the early stage of disease, between surgery or primary systemic therapy. As suggested by Curigliano et al. (15), these two approaches should be considered based on local health-care resources and facilities, numbers of hospital accesses as well as the treatment-related effect on the immune system (16-19). Such decisions do not have a universal value because each situation might differ in a specific country and, also, in a peculiar town or hospital. This highlights even more the role of the multidisciplinary approach within the Institutional Breast Cancer Management Team in order to define the best treatment planning for "that" patient in "that" specific clinical contest. The main goal is to maintain a high quality of care while minimizing COVID-19 infection and treatment-related risks.

Last but not least, the pandemic scenario should be taken into account (i.e., early spread of COVID-19 infection onset as compared to mitigation or recovery phase). Therefore, although in the pre-COVID-19 era an early breast cancer patient might have been treated with primary systemic therapy, in the post-COVID-19 one surgery would be preferably adopted mostly if BCS might be performed in an outpatient setting. The opposite may be true in a postmenopausal patient with an early Luminal A/B disease who could undergo primary endocrine therapy instead of immediate surgery in order to delay hospitalization for more invasive procedures until the end of Covid-19 pandemic.

Regarding the psychological implications of COVID-19 disease, the fear of this new infective disease was perceived by patients as even worse than the diagnosis of cancer. As a matter of fact, in the recent clinical experience by Vanni et al. (20), both patients with suspected breast lesions or confirmed breast cancer assessed in the COVID-19 pandemic showed a significant higher rate of procedure refusal and surgical refusal as compared to the pre-COVID-19 period ( $p=0.0208$, $p=0.0065$ respectively) and this was mostly related to the infection risk. Hence, COVID-19-related anxiety could negatively interfere with the patients' decision-making process, and the availability of such data confirming the safety for both patients and HCWs may prevent any undue delay of treatment with an adverse effect on patient outcome.

\section{Conclusion}

Overall, this validation assessment confirmed that, thanks to the perspective application of this preventive program during this period of dedicated surgical activity, no COVID-19 infection was observed among patients and HCWs; hence, breast cancer surgery can be safely and electively performed while preserving the patient's and HCW's health status without any negative impact on the oncologic outcome.

\section{Conflicts of Interest}

This study received no grant. Matteo Lambertini acted as a consultant for Roche and Novartis, and received honoraria from Theramex, Takeda, Roche, Lilly, Pfizer and Novartis outside the submitted work. All remaining Authors declare no conflicts of interest regarding this study.

\section{Authors' Contributions}

Piero Fregatti: Study planning, surgical treatment, manuscript editing; Marco Gipponi: Study planning, surgical treatment, manuscript editing; Maria Giacchino: Study planning, manuscript editing; Raquel Diaz: Data management (updated); Emanuela Fioravanti: Data management (updated); Benedetta Conte: Study planning; Matteo Lambertini: Study planning; Gabriele Zoppoli: Study planning; Marco Sparavigna: Follow-up and data management; Federica Murelli: Surgical treatment, follow-up and data management; Maria Luisa Toni: Dedicated nurse assistance and data management; Maria Teresa Calabrò: Dedicated nurse assistance and data management; Lina Orsino: Dedicated nurse assistance and data management (updated); Daniele Friedman: Study planning, surgical treatment, manuscript editing.

\section{References}

1 Dipartimento della Protezione civile: COVID-19 Monitoraggio della situazione. Available at: http://www.salute.gov.it/portale/ nuovocoronavirus/dettaglioNotizieNuovoCoronavirus.jsp?lingua= 
italiano $\&$ menu $=$ notizie $\& \mathrm{p}=$ dalministero $\& \mathrm{id}=4875$ [Last accessed on November 17, 2020]

2 Coronavirus Worldwide Graphs. Available at: https:// www.worldometers.info/coronavirus/worldwide-graphs/\#totalcases [Last accessed on November 17, 2020]

3 Coronavirus Worldwide Graphs. Available at: https:// www.worldometers.info/coronavirus/worldwide-graphs/\#totaldeaths [Last accessed on November 17, 2020]

4 The Policlinico San Martino Hospital Center. Available at: http://www.senonetwork.it/centri/dettaglioCentro/74 [Last accessed April 30, 2020]

5 Fregatti P, Gipponi M, Giacchino M, Sparavigna M, Murelli F, Toni ML, Calabrò MT, Orsino L and Friedman D: Breast cancer surgery during the COVID-19 Pandemic: An observational clinical study of the Breast Surgery Clinic at Ospedale Policlinico San Martino - Genoa, Italy. In Vivo 34(3 Suppl): 1667-1673, 2020. PMID: 32503827. DOI: 10.21873/invivo.11959

6 Pneumonia Cases Caused by a Novel Coronavirus, Wuhan, China, 2020. Available at: https://www.ecdc.europa.eu/sites/ default/files/documents/Risk\%20assessment $\% 20-\% 20$ pneumonia \%20Wuhan\%20China\%2017\%20Jan\%202020.pdf [Last accessed on November 17, 2020]

7 Zhou F, Yu T, Du R, Fan G, Liu Y, Liu Z, Xiang J, Wang Y, Song B, Gu X, Guan L, Wei Y, Li H, Wu X, Xu J, Tu S, Zhang $\mathrm{Y}$, Chen $\mathrm{H}$ and $\mathrm{Cao} \mathrm{B}$ : Clinical course and risk factors for mortality of adult inpatients with COVID-19 in Wuhan, China: A retrospective cohort study. Lancet 395: 1054-1062, 2020. PMID: 32171076. DOI: 10.1016/S0140-6736(20)30566-3

8 Long LQ, Huang T, Wang YQ, Wang ZP, Liang Y, Huang TB, Zhang HY, Sun W and Wang T: COVID-19 patients' clinical characteristics, discharge rate and fatality rate of meta-analysis. J Med Virol 92: 577-583, 2020. PMID: 32162702. DOI: $10.1002 /$ jmv. 2575

9 Zhang JJ, Dong X, Cao YY, Yuan YD, Yang YB, Yan YQ, Akdis CA and Gao YD: Clinical characteristics of 140 patients infected with SARS-CoV-2 in Wuhan, China. Allergy 75(7): 1730-1741, 2020. PMID: 32077115. DOI: 10.1111/all.14238

10 COVID 19: Elective Case Triage Guidelines for Surgical Care: Breast Cancer Surgery. Available at: https://www.facs.org/covid19/clinical-guidance/elective-case/breast-cancer [Last accessed on November 16, 2020]

11 WHO - Laboratory testing for 2019 novel coronavirus (2019$\mathrm{nCoV}$ ) in suspected human cases Available at: https:// www.who.int/publications-detail/laboratory-testing-for-2019novel-coronavirus-in-suspected-human-cases-20200117 [Last accessed on November 16, 2020]

12 Sanitaria, Circolare Prot. $\mathrm{N}^{\circ}$ 0001997-22/01/2020-DGPREDGPRE-P del 22 gennaio 2020 All. 3 Polmonite da nuovo coronavirus (2019-nCov) in Cina. Available at: http:// www.trovanorme.salute.gov.it/norme/renderNormsanPdf?anno $=2$ $020 \& \operatorname{codLeg}=72796 \&$ parte $=1 \% 20 \&$ serie $=$ null $[$ Last accessed on April 30, 2020]
13 Sanitaria, Aggiornamento Circolare polmonite 2019-nCoV Prot. $\mathrm{N}^{\circ}$ 0002302-27/01/2020-DGPRE-DGPRE-P All. 1 (A01) del 27/01/2020 Definizione di caso per la segnalazione. Available at: http://www.trovanorme.salute.gov.it/norme/renderNormsan Pdf?anno=2020 \&codLeg=72847\&parte=1\%20\&serie=null [Last accessed on November 16, 2020]

14 Sanitaria, Aggiornamento Circolare polmonite 2019-nCoV Prot. $\mathrm{N}^{\circ}$ 0002302-27/01/2020-DGPRE-DGPRE-P All. 2 (A02) del 27/01/2020 Diagnostica di Laboratorio. Available at: http://www.trovanorme.salute.gov.it/norme/renderNormsanPdf?a nno $=2020 \&$ codLeg $=72847 \&$ parte $=1 \% 20 \&$ serie $=$ null [Last accessed on April 30, 2020]

15 Curigliano G, Cardoso MJ, Poortmans P, Gentilini O, Pravettoni G, Mazzocco K, Houssami N, Pagani O, Senkus E and Cardoso F: Recommendations for triage, prioritization and treatment of breast cancer patients during the COVID-19 pandemic. Breast 52: 8-16, 2020. DOI: 10.1016/j.breast.2020.04.006

16 NHS. Clinical. Guide for the management of cancer patients during the coronavirus pandemic. 2020. Available at: https://www.england.nhs.uk/coronavirus/wpcontent/uploads/sites /52/2020/03/Specialty-guide_Cancer-and-coronavirus_17March.pdf [Last accessed on November 16, 2020]

17 ASCO. Care of individuals with cancer during COVID-19. 2020. Available at: https://www.asco.org/asco-coronavirus-information/ care-individuals-cancer-duringcovid-19 [Last accessed on November 16, 2020]

$18 \mathrm{ASBrS}$. Recommendations for prioritization, treatment and triage of breast cancer patients during the COVID-19 pandemic: executive summary. 2020. Available at: https://www. breastsurgeons.org/docs/news/The_COVID-19_Pandemic_ Breast_Cancer_Consortium_Recommendations_EXECUTIVE_S UMMARY.pdf?01 [Last accessed on November 16, 2020]

19 ECCO. Statement on COVID-19 from the European cancer organisation's board of directors. 2020. Available at: https://www.ecco-org.eu/Global/News/Latest-News/2020/ 03/NEWS-Statement-on-COVID-19-from-the-European-CancerOrganisation-Board-of-Directors [Last accessed on November 16, 2020]

20 Vanni G, Materazzo M, Pellicciaro M, Ingallinella S, Rho M, Santori F, Cotesta M, Caspi J, Makarova A, Pistolese CA and Buonomo OC: Breast Cancer and COVID-19: The effect of fear on patients' decision-making process. In Vivo 34(3S): 16511659, 2020. PMID: 32503825. DOI: 10.21873/invivo.11957

Received October 15, 2020

Revised November 23, 2020

Accepted November 24, 2020 